\title{
Les engagements de la sociolinguistique: Ie cas colombien
}

\author{
(Conditions sociolinguistiques pour la formulation d'une politique linguistique ${ }^{1}$ \\ en accord avec la réalité sociale colombienne) ${ }^{2}$
}

\author{
Miguel Angel Mahecha B. \\ Docente Programa de Lengua Castellana \\ Facultad de Educación \\ mamahechab@usco.edu.co
}

«Il savait que Josiane avait commencé à écrire des livres, il l'avait su par Carol qu'il avait revue à Paris. Et puis il n'avait plus su quoi lui dire. Et puis il le lui avait dit. Il lui avait dit que c'était comme avant, qu'il l'aimait encore, qu'il ne pourrait jamais cesser de l'aimer, qu'il l'aimerait jusqu'à sa mort. "

Résumé

$\mathrm{O}$

$\mathrm{n}$ a dit que la sociolinguistique devrait être un " instrument d'analyse et un moyen de revendication des minorités linguistiques ". À partir du mémoire de Maîtrise d'un chercheur colombien, nous allons présenter une réflexion sur quelques aspects "politiques « actuels en sociolinguistique. Estce que la théorie scientifique se mêle à la réalité sociale ? Peutêtre.

Mots clés : sociolinguistique, politique linguistique, réalité sociale, glottopolitique, Colombie.

\section{Préambule}

Ce document est le produit d'une aventure personnelle qui a commencée à la fin des années 90. Cette aventure (je pense à Barthes) s'est jouée pour moi en deux moments : Le premier moment a commencé avec le travail

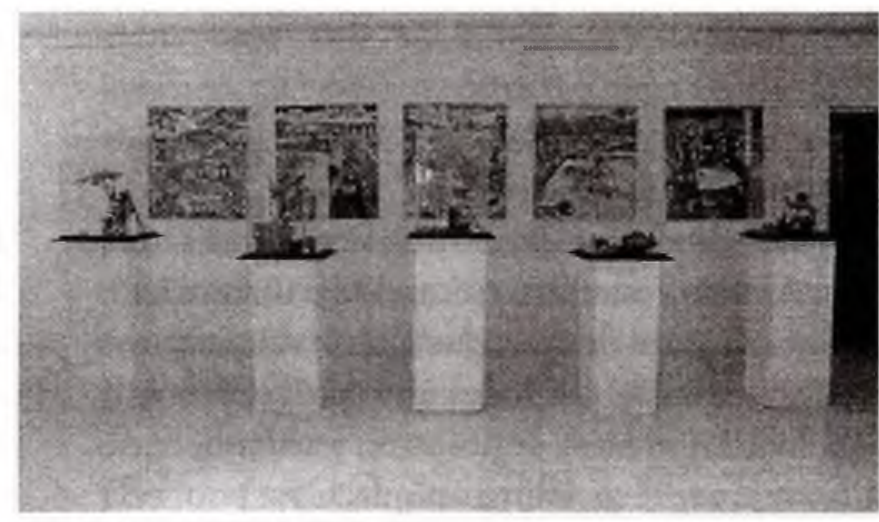

MAGALY CERQUERA SAENZ - "SERIE UN SUSURRO EN EL SILENCIO". PINTURA INSTALACIÓN - Neiva

pionnier à l'Université du Quindio sous la direction de M. Armando Rico quand on a mis au point les thèses d'Hagège (1996). Le deuxième moment a été en 2006, à l'Université Surcolombienne Neiva, quand on a commencé à esquisser un modèle théorique glottopolitique afin d'analyser d'une part, le Programa Nacional de Bilingüismo et d'autre part proposer une politique écolinguistique colombienne. Au

'Et par conséquent une planification linguistique. Pour plus de détails, voir Calvet, 1987 et 1993b.

'Cet article est la version remaniée d'un avant-projet de recherche présenté à l'Lniversité de Rouen en 1997 pour l'inscription au DEA en sociolinguistique. Mes renerciements vont à $M$. Jean-Baptiste Marcellesi et à M. Bernard Gardin pour leurs critiquess très pertinentes et trés précises qui m'ont aidẻ à améliorer ma réflexion. De la mème maniète, je remercie mon ani Gabriel Rueda Olier qui a lu ce manuscrit et n'a apporté des éclaircissensents précieux. 
terme de ce parcours, le lecteur aura pu mesurer la portée d'un modèle socioécolinguistique qui reflète la réalité sociale et linguistique de la Colombie.

Tout d'abord, on suppose que l'on est d'accord sur le fait que l'aspect politique et son incidence sur les recherches linguistiques constitue un des objets de la sociolinguistique ${ }^{3}$. Selon Armando Rico, on peut diviser les approches sociolinguistiques en deux domaines : "Parmi tous les domaines de recherches relevant de la sociolinguistique deux types d'étude peuvent les regrouper : a) les études portant sur la variation linguistique d'une même langue selon les groupes sociaux, b) celles qui portent sur le problème de conflit linguistique dans des situations de multilinguisme, bilinguisme diglossie, langues en contact dans une ou des communautés déterminées.» ${ }^{4}$

De nombreuses études ont été faites par des linguistes et des sociologues afin de montrer l'influence $\mathrm{du}$ pouvoir politique dans des diverses manifestations culturelles d'une société. À travers l'usage de la langue -plutôt du discourson est arrivé à imposer des préceptes qui débouchent sur une conception particulière de la communauté sociolinguistique. C'est pour cela que l'on a étudié sous l'optique sociolinguistique le discours politique, le discours en situation de travail, les discours constituants, bref, tous les discours visant le pouvoir ou engagés dans sa conquête ${ }^{5}$.

La sociolinguistique a tenté d'établir au cours des années ${ }^{6}$ un objet d'étude vaste et embrouillé?
Autrement dit, elle veut rendre compte de la plupart des phénomènes sociaux qui cohabitent avec le lexème « langue « en se mêlant des autres visions (i.e. approches, méthodes, modèles, etc.) telles que la sémiotique, la ethnométhodologie, la psychologie, l'anthropologie, la psychologique, etc. Aujourd'hui la sociolinguistique est un amas de visions divergentes qui ne cesse pas de se multiplier et déclenche des vides à l'intérieur de la réflexion scientifique quand on veut se pencher sur les rapports langue-société, si tant est qu'ils puissent exister!

On sait que les tendances actuelles de la linguistique poussent à la reformulation, précisément de son objet d'étude ${ }^{8}$. La sociolinguistique en est une qui considère sérieusement les aspects «externes» qui entourent la langue. En d'autres termes, elle n'est qu'une linguistique de la parole :

[...] c'est-à-dire une linguistique qui, sans négliger les acquis de l'approche structuraliste des phénomènes langagiers, situe son objet dans l'ordre du social et $d u$ quotidien, du privé et du politique, de l'action et de l'intéraction, pour étudier aussi bien les variations dans l'usage des mots que les rituels de conversation, les situations de communication que les institutions de la langue, les pratiques singulières de langage que les phénomènes collectifs liés au plurilinguisme[...] (Boyer, 1996b, p. 6).

Force est de reconnaître qu'il n'y a pas d'accès unique à l'étude des langues et leurs rapports intimes avec la réalité sociale, mais un éventail d'approches conduites par le désir de tout

\footnotetext{
'L'aspect politique a èté traité conme une espece de variable sociolinguistique. Après, il a été étudié par les analystes du discours et les chercheurs qui s'uccupent de l'anénagenent linguisticque.

${ }^{4}$ Armuando Rico, 1981, pág. 1

'Ct. la présentation de Doninique Maingueneau à la revue LANGAGES 117.

"Les origines épistémologic|ues de la sociolinguistique sont obscures. Nous renvoyons les lecteurs aux textes sources de Gardin et Marcellesi, 1974 et 1980.

"Considérez ces deux propositions: "La scciolinguisticue n'a pas un objet rigoureusentent défini et les épisténologues ont peut ẻtre raison de faire la fine bouche. "(Marcellesi, $1980,11)$ et " L'objet d'étude de la linguistique n'est pas seulentent la langue ou les langues mais la coninunaute sociale sous son aspect linguistique. De ce point de vue, il n'y a plus lieu de distinguer entre sociolinguistique et linguistiçue... " (L.-J. Calvet, 1993b, 109).

"Corrélativenent le terme de langue peut, s'il est défini conıne le répondant objectif du systène des jugements granmaticaux, être rendu indépendant de l'inportance suciohistoricute des locuteurs. "(J.C. Milner, 1989,60) L'objet d'étude de la linguistique est-il le langage ou la langue ! Cf. Mahecha 1999 (inédit).
} 
expliquer. La sociolinguistique illustre précisément ce trait et devient un domaine qui embrasse les plus diverses visions englobant d'une part, les variétés sociales, les attitudes des locuteurs, le contexte, le discours, les politiques linguistiques, etc., et d'autre part, les aspects morphosyntaxique et phonologique d'une langue. De cette façon on croit intégrer linguistique interne et linguistique externe (c.f. Calvet, 1981, p. 15-20).

Considérons encore le problème de l'objet d'étude. Les éditeurs du Cours de linguistique générale de Ferdinand de Saussure faisaient au début du XXe siècle une affirmation bouleversante qui malgré toutes les proliférations théoriques ${ }^{9}$, continue en vigueur: Bien loin que l'objet précède le point de vue, on dirait que c'est le point de vue qui crée l'objet, et d'ailleurs rien ne nous dit d'avance que l'une de ces manières de considérer le fait en question soit antérieure ou supérieure aux autres. (CLG, 1964, p. 23).

Dans ces conditions on est en mesure de dire que nous n'arriverons jamais à formuler "le " objet d'étude de la linguistique car celui-ci n'existe pas. Ce qui existe c'est le système ensemble- d'objets possibles (présentés sous la forme de morphèmes tels que socio-, ethno-, pragma, bio-, sémio-, etc.) d'où se dégage la sociolinguistique. Toutefois, l'approche dite sémiotique essaie d'intégrer les diverses visions et tend vers la présentation du langage -la faculté biologique-comme le sommet sémiotique qui les englobe : "L'objet des sciences sociales ne peut être que sémiotique. Un fait n'est psychologique, historique, social ou linguistique que pris dans le sens, c'est-à-dire, de façon directe ou indirecte, dans le langage. "(Achard, 1995, p. 83).

Bien que l'on ait besoin d'une approche épistémologique rigoureuse ${ }^{10}$ pour mettre un peu d'ordre dans ce foisonnement théorique et empirique, notre but dans les lignes qui suivent s'écarte d'une telle démarche, Hélas ! Nous partons du fait, plutôt du principe que la sociolinguistique est une " discipline militante et sa tâche doit contribuer au développement de la lutte pour une politique linguistique bouleversante : (A. Rico, 1981, introduction). Nous allons considérer la "réflexion sociolinguistique " comme un véhicule pour arriver à décrire -i.e. identifier- les plus variées politiques linguistiques ${ }^{11}$ qu'un pouvoir de l'Etat -dans notre cas le colombien- peut imposer, voire implanter. C'est dans cette perspective que nous regardons.

Nous ferons référence au Mémoire de Maîtrise d'Armando Rico O., intitulé "Problèmes sociolinguistiques de la société colombienne ", soutenu à l'Université de Grenoble III en 1981. A partir de ce travail qui dénonce l'abus des pouvoirs (partis) politiques et le sacrifice des indigènes en Colombie, on va considèrer l'engagement de la sociolinguistique dans la réalité sociale colombienne au XXIe siècle.

Un dernier mot. Cet article prétend d'une part, faire connaitre un des travaux d'Armando Rico à propos de la sociolinguistique colombienne et d'autre part, lui rendre un hommage après sa mort absurde : "En paz descanse esta vida tocada

\footnotetext{
"Les diverses opinions qui se dėgagent de la pensėe "écrite" saussurienne. Le CLG est publié après la nort de Ferdinand de Saussure et les inconsistances théoriques voire methodologiques n'ont pas pu disparaitre à cause de l'absence des jugenents néticuleux du Maître. "Conscients de la difficulté de transposition par écrit d'un texte dit, ils renoncent à tout publier. Ils rejettent également l'option qui aurait consisté à n'éditer qu'un seul des cours, car lé programne suivi n'était pas le méme d'une année sur l'autre, et des élénents intéressants auraient été perdus; ou à n'éditer que des norceaux choisis, ce qui aurait occulté tout développenent suivi. Ils adoptent une solution 'plus hardie' ("tenter une reconstitution, une synchèse, sur la base du troisiène cours, en utilisant tous les natériaux dont nous disposions"), tout en étant conscients des linsites de leur entreprise " (F. Gadet, 1990,18).

${ }^{10} \mathrm{Cf}$. Mahecha, 1999, Gardin et Marcellesi, 1980 et Calvet 1974 et $19993 \mathrm{~b}$.

"Et par conséçuent les planifications linguistiques étayées par le glottopolitique.

1'I He pensée qui fait le bilan de la vie d'Amando. Voila l'épitaphe dont l'auteur est le professeur Rubein Arboleda.
} 
por la aflicción de la estrechez y la ansiedad de la amplitud abrazada." 12

1. La Colombie

\subsection{Situation physique}

La Colombie est un pays situé au Nord-Ouest de l'Amérique du Sud. Elle a pour voisins cinq pays : le Venezuela, à l'Est, l'Equateur et le Pérou au Sud et le Brésil au Sud-est. De la même manière, elle limite au nord avec l'Océan Atlantique et à l'ouest avec l'Océan Pacifique. Cette situation géostratégique lui permet d'être l'union entre l'Amérique centrale et l'Amérique du sud.

Ses 1' 138.914 kilomètres carrés s'étendent de $12^{\circ} 30^{\prime} 40^{\prime \prime}$ de latitude nord (la Guajira) à $4^{\circ} 13^{\prime}$ $30^{\prime \prime}$ de latitude sud où l'on trouve Leticia, un port sur l'Amazone. La Colombie est l'unique pays sud-américain à bénéficier des deux côtes maritimes : l'une d'elles s'ouvre sur les Caraïbes et l'autre sur le Pacifique. La Colombie possède plusieurs îles : L'Archipel de San Andrés et Providencia, Gorgona, Gorgonilla et Malpelo. Trois fleuves dominent l'hydrographie du pays: le Magdalena $(1550 \mathrm{Km})$, son affluent le Cauca $(1350 \mathrm{Km})$ et l'Atrato $(750 \mathrm{Km})$.

Le relief colombien est très fragmenté. La chaîne des Andes se divise à l'intérieur du territoire colombien en trois branches : cordillère occidentale, cordillère centrale et cordillère orientale. Celle-ci s'élargit pour configurer deux branches, l'une d'elles vers là Guajira, l'autre vers le Venezuela à travers la cordillère de Merida. Il y a une grande quantité des volcans et des montagnes (la Colombie appartient à la ceinture du feu du Pacifique). Les sommets les plus élevés son le Mont Nevado del Huila (5365m) et le Pic Colon ( $5775 \mathrm{~m}$ ).

L'Agglomération colombienne actuelle compte près de 47'000.000 millions d'habitants. La Colombie a une densité moyenne de 37 habitants au $\mathrm{Km}^{2}$; de plus, la population est inégalement repartie. Le pourcentage le plus élevé se trouve dans les Andes ( $30 \mathrm{hab} . / \mathrm{Km}^{2}$ ), le plus faible se trouve dans les plaines orientales ( 3 hab. $/ \mathrm{Km}^{2}$ ). L'éventail culturel colombien est riche et il peut s'illustrer par une description anecdotique colombienne :

Habitué aux paisibles changements de saisons des changements qui se déroulent dans le temps et non dans l'espace- un Européen a du mal à imaginer le violent contraste qui, dans un même pays peut exister entre le monde des Caraïbes et le monde des Andes. Un contraste géographique d'abord. Monde de lumières et de chaleur, celui des caraibes ne pourrait se peindre qu'en bleus et verts profonds. Monde de brumes, de pluies fines et de bises, celui des Andes déploie une gamme délicate de gris et des verts mélancoliques. Contraste humain aussi. Fils d'Andalous, des Noirs et d'arrogants indiens caraïbes, l'homme de la côte est ouvert, exubérant, étranger à toute solennité, rebelle à toute hiérarchie et à tout protocole. Il aime la danse; des rythmes africains, percutants, survivent dans sa musique. Au contraire, le colombien de la cordillère marqué par le formalisme castillan et par le tempérament taciturne et méfiant de l'indien Chibcha, est un homme subtilement réservé et cérémonieux; dans son humour aussi il y $a$ de la sensibilité. La courtoisie de ses manières peut masquer un fond d'agressivité que l'alcool fait parfois sortir au grand jour de façon intempestive. (La violence politique n'a jamais partie de la côte, toujours $d u$ haut pays).Comme le paysage qui environne l'homme andin, la musique de ce demier est plaintive : elle parle de l'abandon, d'absences, d'amours qui s'envolent. ${ }^{13}$

\subsection{Bref aperçu historique}

Pour mieux illustrer la situation sociolinguistique colombienne, on va présenter quelques événements historiques pris dans leur ensemble.

"I Ine odeur de goyave, 1982. 
Tout d'abord, si l'on considère l'avis de $\mathrm{M}$. Minaudier dans son histoire de la Colombie ${ }^{14}$, quant aux événements colombiens, on peut affirmer qu'ils ont été discrets sur l'échiquier international, mais ils peuvent et doivent être abordés :

L'histoire de la Colombie est une histoire périphérique, effacée. Discrète sur la scène internationale, la Colombie a plus reçu qu'elle n'a donné, plus subi qu'elle n'a influencé. Elle a fourni peu de grandes figures de stature internationale, à l'exception de Bolivar (d'ailleurs vénézuélien de naissance), et comme elle n'a pas abrité de grand empire précolombien, c'est un pays peu touristique, donc peu connu. Il y a peu de colombiens en France, peu de liens entre la France et la Colombie ; aussi ce pays a-t-il attiré peu les chercheurs français. Pourtant la Colombie ne mérite pas cette méconnaissance. Elle dispose d'un fabuleux patrimoine naturel et culturel ; son histoire est d'un grand intérêt, depuis l'époque de la Conquête (c'es là qui naquit le mythe de l'Eldorado) jusqu'aux convulsions de l'époque actuelle (le pays est au centre de la crise de la drogue, un phénomène aux enjeux mondiaux). La Colombie est un bon exemple de société multiraciale et métisse, marquée par la violence mais aussi par une intégration des communautés bien plus forte qu'au Pérou par exemple ; c'est aussi un très bon modèle de constitution progressive d'une nation à partir d'un ensemble de régions très diverses, mal reliées entre elles, souvent antagonistes.

Cependant n'importe quel fait historique peut se révéler important et décisif quand on veut comprendre voire dessiner l'image d'un territoire. Un fait historique est un fait de culture, c'est-à-dire, il est la représentation d'un exercice mental de la part d'une collectivité. En ce qui concerne la Colombie, l'histoire révèle que le territoire actuel n'a été le siège d'aucun empire comparable à celui des Aztèques, des
Mayas ou des Incas. Les cultures qui s'y épanouissent prospèrent dans des zones géographiques limitées.

Après la Conquête, les conquistadores se sont mis à exploiter les richesses du territoire, en particulier l'or et les émeraudes. Pour ce faire, les indiens sont obligés à travailler dans les mines et l'abus produit un vrai carnage. Après la conquête sanglante vient la Colonie où l'on pratique l'esclavage de noirs venus de l'Afrique. L'histoire de la Colombie se fond dans celle de l'Amérique espagnole jusqu'à l'heure de l'indépendance le 7 août 1819 avec la victoire de Bolivar au Pont de Boyacá. Cependant le pouvoir arraché aux espagnols devient l'apanage exclusif des créoles (criollos). En ce qui concerne la situation des noirs et des indiens, elle ne se trouve guère améliorée.

Au cours du XIXe siècle, la vie du pays va être marquée par l'affrontement de deux partis politiques : le parti libéral et le parti conservateur. Cet affrontement va devenir la cause de multiples conflits et guerres civiles qui déchirent petit à petit la Colombie. En 1886 on proclame la Constitution politique de la Colombie dont les auteurs sont Rafael Nuñez et Miguel Antonio Caro. En 1903 les Etats-Unis s'approprient de Panama et construisent le Canal. En 1948 le leader libéral Jorge Eliecer Gaitán fut assassiné et sa mort a déclenché non seulement une émeute, le Bogotazo mais aussi l'époque de la Violence. Au cours des années soixante et soixante dix les guérillas font leur apparition (FARC, ELN, M-19)

Armando Rico ajoute,

Dans le cas de la colonisation espagnole, l'imposition linguistique se fait dès le début et non pas quelque temps après l'invasion. Avec l'arrivée des premiers conquérants, on a remarqué la présence des missionnaires qui avaient pour tâche non seulement

14].P. Minaudier. 1992. Histoire de la Colombie. De la conquête à noss jours. Paris, Editions L'Harnattan. 
d'imposer la religion chrétienne mais aussi d'imposer la langue espagnole. Leur mission dans le Nouveau monde a été d'accomplir la tâche des conquérants quand ils sont impuissants avec les armes; les missionnaires au nom de Dieu et avec la croix et la langue comme des armes ont réussi à mettre des tribus indomptées sous le joug de la couronne espagnole. Les missionnaires ont enseigné la langue espagnole aux indigènes de l'Amérique para la religion. La religion et la langue se mélangent toujours au service du pouvoir. ${ }^{15}$

Ces idées ne sont nullement invraisemblables. Ce qui importe pour l'instant, c'est préciser quelques affirmations pour éviter l'imprécision historique. Par souci d'exactitude, nous allons ajouter l'avis démystificateur de M. Triana y Antorveza, en tant que spécialiste de l'histoire colombienne :

Como toda labor cientifica, también la historia parte de hipótesis de trabajo que el proceso investigativo debe confirmar o rechazar. En el presente caso, la hipótesis fundamental fue la siguiente: España al colonizar estos teritorios impuso la lengua castellana, destruyendo sin fórmula de juicio nuestras lenguas indigenas. La respuesta histórica obtenida mediante el empleo de la metodologia de la técnica investigativa descarta la hipótesis anterior, aunque se reconocen diferentes instancias:

a) El Estado español mantuvo una actitud prudente frente a las lenguas vernáculas, aunque su posición fue oscilante en ocasiones en razón de las discusiones locales.

b) Ante la imposibilidad de que los doctrineros pudieran aprender todas y cada una de las lenguas nativas, España, siguiendo antiguas costumbres de los incas y de los aztecas, les confirió a algunas el carácter de lenguas generales para que sirvieran como instrumentos teológicos. Sin esta actitud, quizás no contariamos hoy con algún acervo documental y bibliográfico al respecto.

c) España antes del descubrimiento se caracterizaba por su dispersión lingüistica, siendo el castellano tan sólo uno de los elementos del mosaico cultural ibérico. América dio la oportunidad para la expansión y universalización del idioma de los Reyes Católicos, el cual también comenzó antes de finalizar 1492 a integrar muchas aportaciones fónicas y léxicas procedentes del pluralismo étnico y cultural de nuestro Continente.

d) Frente a la atomización cultural y lingüistica de nuestro pais, correspondió al castellano desempeñar el papel de elemento idiomático unificador, atemperado por la conciencia juridica de la Corona y su enfoque religioso, aunque no siempre resultó fácil de poner esto en práctica.

e) Como el problema del idioma inquietó tempranamente a conquistadores, colonos, hombres de Iglesia y gobernantes, se creó todo un corpus juridico-lingüistico que le dio prelación a las lenguas locales en aras de la evangelización, siendo política real que tuvo vigencia desde Felipe II hasta Carlos III, rey éste último que pensó en forma diferente y actuó para su epoca según su concepción ilustrada. ${ }^{16}$

Bien que la Colombie n'ait apporté des crises sociales bouleversantes pour l'histoire universelle, (actuellement elle est le noyau, voire l'épicentre des luttes politiques, d'un conflit social interne, l'enlèvement, la parapolitique, l'abus du pouvoir de la part du président Uribe, le conflit diplomatique avec le Venezuela et l' Equateur, les bases militaires des américains dans le sol colombien, etc.) le peuple colombien cultive l'amour pour une patrie qui se réclame $\mathrm{du}$ sol américain, un vertigineux retour aux sources!

\footnotetext{
"A. Rico, 1981, p.

1"Triana y Antorveza, 1987, p. XV.XVI.
} 


\subsection{Esquisse de description sociolinguistique}

La population colombienne est conformée par des apports ethnogénétiques différents : des blancs, des indiens, des noirs. C'est un métissage authentique et complexe. C'est pour cela que l'on trouve actuellement un éventail linguistique riche et représentatif : l'espagnol -avec ses variations dialectales, langue officielle dans tout le territoire national, 64 langues indigènes, deux langues créoles, l'un d'eux, de base anglaise se trouve à l'Archipel de San Andrés et l'autre, de base espagnole se troụve à San Basilio de Palenque.

Pour ce qui est des langues colombiennes que l'on pourrait définir langues ethniques ${ }^{17}$, elles sont respectées para la Constitution Politique de 1991. C'est très beau en théorie mais en fait il en est autrement :

ARTICULO 7: Minorias raciales y culturales. El Estado reconoce y protege la diversidad étnica y cultural de la Nación colombiana.

ARTICULO 10: Idiomas. El castellano es el idioma oficial de Colombia. Las lenguas y dialectos de los grupos étnicos son también oficiales en sus territorios. La enseñanza que se imparta en las comunidades con tradiciones lingüisticas propias será bilingüe.

L'espagnol en Colombie a une place privilégiée. Cette langue n'est pas seulement la langue officielle du pays mais aussi la langue qui s'impose de manière naturelle. Ce trait tend à représenter soit directement, soit indirectement un phénomène de glottophagie que nous traiterons plus loin. De plus, l'espagnol véhicule un potentiel culturel énorme qui nous lie à l'Espagne et à la communauté hispanophone mondiale et, d'une certaine manière, tend à nuire aux autres langues (les langues créoles et les langues indigènes) qui essaient de survivre.
Il faudrait proposer une politique linguistique en accord avec la réalité sociale colombienne afin que l'espagnol puisse partager son rôle sociolinguistique avec les langues minoritaires. Les deux créoles enrichissent de la même manière que les langues indigènes le panorama linguistique du pays. Ce sont des variétés créoles en raison de leur genèse et de leurs traits de structure (à ce sujet voir Patiño Rosselli 1983, 1996 et Forbes 1987).

Le créole de San Andres (Bendé) a été lexifié par l'anglais et s'est développé dans ces îles au cours du XVIII siècle, parmi une population venue de la Jamaĩque. Le créole de base espagnole, le palenquero, est l'unique qui existe aujourd'hui en Amérique. Son domaine se trouve à San Basilio de Palenque, un petit village demeuré une espèce d'îlot afroaméricain qui s'est imposé a la surface « costeña " (c'est-a-dire, de la côte atlantique) qui l'a entouré. La culture de ce village est encore aujourd'hui presque intégralement africaine. Grâce à un programme d'ethnoéducation sous les auspices du Ministère de l'Education Nationale, on est arrivé à stimuler l'utilisation du créole palenquero dont la communauté peut et doit être fière. De plus, celui-là est présent dans l'enseignement primaire et secondaire.

À San Andres le créole bendé a de temps en temps une fonction véhiculaire. Il est considéré une variété basse de l'anglais standard et, malgré son utilisation fréquente -et c'est la langue maternelle de la plupart de la population-c'est l'espagnol la langue qui véhicule les fonctions officielles. Bien que les locuteurs de San Andres communiquent aisément en espagnol et en anglais, ils conservent leur créole comme une marque d'identité sociolinguistique.

Pour ce qui est des langues indigènes, la situation est pareille. Elles sont considérées langues officielles dans leurs territoires mais subordonnées par la fonction officielle-

${ }^{12}$ Lanques parlèes para des groupes restreints. C'est le cas des langues indigènes et les crèoles palenquero et bendė. 
nationale-véhiculaire de l'espagnol. Le professeur Armando Rico a suffisamment illustré ces aspects-là. Les recherches scientifiques sur les langues indigènes se développent actuellement avec une intensité notable ${ }^{18}$.

2. L'Espagnol : moyen de domination et véhicule de pouvoir

Nous allons d'une part, poser à nouveau le cadre théorique proposé par le professeur Armando Rico dans le mémoire cité supra et d'autre part, essayer de décrire l'aménagement linguistique colombien sous la bannière de la sociolinguistique.

\subsection{Glottophagie réussie en Colombie?}

Ce qui caractérise la Colombie c'est que la langue officielle, l'espagnol est largement dominant et il n'y a pas sur le territoire d'autres langues qui puissent la remplacer voire la déplacer. Pour pouvoir répondre à la question posée ci-dessus, il faut, d'un côté que l'on précise quelques concepts sociolinguistiques qui ont été au cours des années problématiques et d'autre part, tenter d'identifier l'idéologie de l'Etat colombien présente dans la conception d'identité du peuple colombien. Considérons maintenant les deux premiers concepts : dialecte et communauté linguistique.

Le terme dialecte a posé beaucoup de problèmes non encore résolus parce qu'il présente un caractère mixte et peut être défini selon les approches qui se réclament soit de la géographie linguistique et la dialectologie, soit de la sociolinguistique, soit de la politique linguistique (avec la variante colonialiste-impérialiste). Pour Coseriu, les dialectes peuvent être primaires (antérieurs à la constitution d'une possible 'langue commune') secondaires (résultat de la différentiation régionale de la langue commune) et, tertiaires (résultant de la différentiation régionale de l'éventuelle langue standard, c'est-à-dire, de l'éventuelle modalité 'exemplaire' de la langue commune). Il faut remarquer donc que la relation existant entre dialecte, niveau de style de langue est une relation dirigée, précisément vers le sens suivant : dialecteniveau-style de langue ; c'est dire qu'un dialecte peut fonctionner comme niveau et comme style de langue et un niveau peut aussi fonctionner comme style de langue mais non à l' envers.

Cette définition nous parait concilier des postulats de la dialectologie, c'est-à-dire d'une géographie linguistique élaborée, et des postulats de la sociolinguistique dans la mesure où l'on traite des facteurs historiques et linguistiques sans faire appel aux relations de pouvoir.

L'étude du dialecte conduit à rendre compte des forces que celui-ci peut prendre dans une isoglosse déterminée (le syntopique, le syntrasthique et le synphasique). Donc, le dialecte représente l'espace, la forme et l'application d'une production langagière naturelle.

Dans la même perspective, M.J.J. Montes, un théoricien de la dialectologie, s'approche de la vision de Coseriu :

El dialecto puede definirse pues asi: Forma idiomática caracterizada por un conjunto de formas que la individualizan frente a otros idiomas o incluida en un conjunto idiomático mayor, ora porque es una derivación histórico-estructural de tal conjunto (aspecto meramente histórico), ora porque además de compartir un núcleo estructural básico con el conjunto mayor, se subordina sincrónicamente a su forma modélica y a su dialecto literario, o bien, por último, porque sin ser parte históricamente de la estructura del sistema excluyente está subordinado a

\footnotetext{
"Vorr le dernier livre sur les langues indigènes publié par l'Institut Caro et Cuervo en 2000.
} 
él para algunas funciones comunicativas y en la norma modélica de máximo prestigio.

Tout d'abord M. Montes considère la dialectologie comme une discipline autonome qui rend compte de la variation à l'intérieur d'une langue historique. Pour ce faire, elle doit s'appuyer sur trois disciplines auxiliaires ${ }^{19}$ : la grammaire ou typologie linguistique, la sociolinguistique et la géographie linguistique. Le dialecte n'est plus une forme isolée à cause de l'attitude péjorative envers les formes particulières de la langue. C'est la manifestation la plus proche du parler naturel d'une communauté. Autrement dit, le dialecte est la langue qui présente un système linguistique/ idiomatique élaboré et qui engendre l'ensemble de normes dont la communauté linguistique dispose. La prétendue pauvreté du dialecte n'existe plus.

Avec la perspective colonialiste- impérialiste, Louis-Jean Calvet est un des rares auteurs qui se sont intéressés à l'influence du politique et du glottophagique sur les aspects linguistiques. ${ }^{20}$ Dans le chapitre 2 de son livre Linguistique et Colonialisme, il analyse la relation entre la langue et les dialectes. La question ne consiste pas à savoir si le dialecte est une forme linguistique avec un statut de langue mais de savoir si cette forme appartient au domaine de la linguistique ou au domaine du politique (social) : « le français n'est pas une langue de toute éternité ou de droit divin, il l'est devenu historiquement à partir du dialecte francien et au cours d'un processus qui n'a rien de linguistique. Mais la littérature linguistique a permis de croire à la validité théorique de cette opposition sur un terrain qui n'était pas le sien.. ${ }^{21}$

Ainsi, le dialecte garde un caractère diachronique. Celui-ci le lie à la définition de «langue» mettant en même temps un peu d'ordre dans les déviations théoriques qui mêlent des facteurs linguistiques et des facteurs sociaux avec une dose d'impérialisme. Il n'y a pas lieu de distinguer entre dialecte et langue parce que du point de vue linguistique ils ont le même statut. Du point de vue (glotto)politique, ce sont deux formes hiérarchiquement différentes.

Calvet insiste :

Tout ce qui précède montre à l'évidence que le dialecte n'est jamais qu'une langue battue, et que la langue est un dialecte qui a réussi politiquement. $\mathrm{Ou}$ plus précisément, un dialecte dont les locuteurs ont pris une certaine forme de pouvoir para la médiation de certaines formes sociales et politiques dans un certain cadre économique [...] Aussi, pour éviter tout risque de mécompréhension n'utiliserons nous plus dans les pages qui suivent le terme dialecte et, lorsque les rapports linguistiques dont nous traiterons seront insérés dans les rapports de force, parlerons-nous uniquement de langue dominée et de langue dominante. ${ }^{22}$

Dans la même direction, Milner (1989), fait observer que :

Il ne s'agit pas de la différence langue/dialecte et il ne s'agit pas de distinguer ce que parmi les formations langagières méritent le nom de langue. Cette question est purement sociologique et n'a pas de statut précis en linguistique. On ne la prendra pas donc en considération. Il s'agit de toute autre chose : distinguer entre ce qui est une formation langagière et ce qui ne l'est pas. Le terme langue est pris alors dans son acception la plus large : langues, dialectes, créoles, etc., en font partie, puisque ce sont tous des formulations langagières, distinguées seulement par des statuts sociologiques différents. ${ }^{23}$

Dans les cas que nous venons de citer, le dialecte

\footnotetext{
(1) En suivant Theban, 1968, 659-663

20 Calvet, 1974

"Op. Cit. page 41

"Op. Cit. page
} 
n'est plus une forme dégradée de la langue, il est par excellence un système linguistique complexe utilisé par une communauté linguistique restreinte qui dans certains cas, n'a pas atteint le rôle politique de langue nationale. Toutefois il faudrait se demander si la question sociopolitique influence de manière légitime les postulats de la science linguistique, ou dans le cas contraire, elle est une déviation théorique produite par une fausse conception de l'observation d'un fait de langue.

En ce qui concerne la communauté linguistique, nous ferons référence à l'excellente approche de M. Calvet ${ }^{24}$. Celle-ci est un parcours théorique parmi les définitions les plus variées de la part des linguistes. M. Calvet critique, d'une part, le point de départ de certains chercheurs pace que ceux-ci ont abordé ce syntagme à partir du linguistique et ont négligé la partie sociale :

"Dans tous les cas, la démarche est la même : on part de la langue (sans toujours la définir) pour définir le groupe." et plus loin, "Ce problème est central car les linguistes, lorsqu'ils veulent définir une communauté linguistique, ne retiennent de ce syntagme que le deuxième terme, l'adjectif, comme si dans communauté linguistique il n'y avait que langue, oubliant qu'il y a aussi communauté." 25

D'autre part, M. Calvet propose une démarche intégrale qui s'occupera non seulement du linguistique mais aussi du social parce qu'en fin de compte, " l'objet de la linguistique n'est pas seulement la langue ou les langues mais la communauté sociale sous son aspect linguistiquen ${ }^{26}$ Considérons donc les postulats de Calvet.

"Une communauté linguistique est constituée par des gens qui ont la même première langue? [...] si l'on répond oui à cette question une des retombées de cette définition serait que, à part quelques cas statistiquement marginaux (les bilingues précoces), un individu ne peut appartenir qu'à une seule communauté linguistique."

Calvet affirme qu'il est tout à fait raisonnable de supposer que le lien commun entre les gens d'une communauté est l'utilisation d'une langue particulière. Toutefois cette déclaration peut être déformée parce que de nombreuses personnes pourraient utiliser dans cette même communauté, deux ou plusieurs langues. Pour mieux illustrer ce cas, considérons la situation sociolinguistique des quatre groupes des locuteurs : colombiens, paraguayens, portoricains et suisses. Pour les premiers, on peut trouver des locuteurs indigènes qui utilisent dans sa réalité deux langues : une langue indigène (p.ex. le Saliba, le Piratapuyo, le Tukano, le Pisamira) qui est sa langue maternelle mais est une langue subordonnée et la langue espagnole. Ce groupe maîtrise les deux langues. Pour le deuxième groupe, les locuteurs utilisent deux langues, l'une d'elles c'est l'espagnol et l'autre, une langue indigène, le guarani et celles-ci sont des langues officielles, c'est-à-dire, elles ont le même statut politique. Pour le troisième groupe, les locuteurs utilisent deux langues qui ont non seulement le même statut politique mais aussi qu'elles sont langues de civilisation, l'espagnol et l'anglais. Finalement, pour le cas du groupe suisse, nous avons des locuteurs qui parlent le français, l'allemand, l'italien et le romanche. Peut-on affirmer que les groupes des locuteurs cités supra, appartiennent-ils à la même communauté linguistique?

De plus, il existe une autre possibilité :

\footnotetext{
"Milner, 1989, page

24 1993b, payes:

25 1993b, pages 87 et 90 .

3c. 1993b, page 90 .
} 
"Une communauté linguistique pourrait être constituée par des gens qui se comprennent grâce à une même langue»; c'est-à-dire, il y aurait des gens qui appartiendraient à plusieurs communautés linguistiques selon le nombre des langues utilisées. Mais où se trouve l'unité et l'appartenance à un groupe voire à une communauté ? Calvet nous conseille : "La seule façon de sortir de ces paradoxes est de sortir de la langue et partir de la réalité sociale. A l'intérieur d'une communauté on peut trouver tout un éventail de composantes dont la langue fait partie. La 'réalité sociale' englobe le linguistique et le social. " En effet, dans un groupe social, il y a bien entendu des locuteurs, des codes, des variétés des codes, des rapports des locuteurs à ces codes et des situations de communications ${ }^{27}$

Finalement, voici les points dont le linguiste, selon Calvet, doit en tenir compte pour décrire les rapports existant à l'intérieur d'une communauté linguistique :

La tâche du linguiste est donc décrire chacun de ces éléments ainsi que leurs rapponts mutuels :

1/Décrire les codes en présence (c'est en gros ce que font les différentes linguistiques), mais en prenant en compte la dimension diachronique, l'histoire de ces codes et des gens qui les utilisent (ce que ne font pas toutes les linguistiques);

2/Structurer la communauté en fonction de ces codes, c'est-à-dire décrire les sous-groupes selon les langues qu'ils parlent, les lieux où ils les parlent, avec qui ils les parlent, pourquoi ils les parlent, etc., décrire aussi les réseaux de communication, les comportements, les attitudes... ;

3/Décrire les variations dans l'usage des codes en fonction des diverses variables sociales (sexe, catégories sociales, âge, etc.);

4/Décrire les effets sur les codes eux-mêmes de cette coexistence : emprunts, interférences, etc. 5/ Décrire les effets sur les codes de la situation sociales : c'est le problème des rapports entre forme et fonction. 28

Revenons-en à la question posée plus haut : Estce qu'il y a eu de glottophagie réussie en Colombie? La réponse ne peut être qu'affirmative. Comme tout phénomène colonial, l'oppression et le déplacement des patrimoines culturels (dont la langue est un des ingrédients) se manifeste sous une imposture idéologique qui se renouvelle sans possibilité de disparaître. Une culture s'impose à une autre et impose sa vision du monde. Un exemple actuel est illustré par les Etats-Unis ; ils ont réussi à imposer sinon la langue, du moins la culture américaine. Malheureusement la langue devient un véhicule de pouvoir qui entraîne une idéologie nocive à la cosmovision du territoire colonisé.

La Colombie et les autres pays de l'Amérique latine ont été colonisés par l'Espagne. Les langues indigènes ont été dévorées par la langue espagnole. C'est le phénomène de glottophagie $^{29}$. Celle-ci représente le premier stade, le colonialisme naissant :

On sait que toute invasion se concrétise très vite para l'implantation de groupes des militaires et d'administrateurs, puis de commerçants, généralement dans les villes: c'est autour de ces groupes que va tout d'abord se jouer la bataille linguistique. Se dégage en effet, comme je le rappelais au début de ce chapitre, une classe de collaborateurs locaux qui vont, par nécessité et par intérêt, utiliser la langue de l'envahisseur. Il s'agit bien sûr tout d'abord de ce que l'on appelle aujourd'hui les bourgeoisies compradores, mais cette fonction a été remplie de tout temps par diverses catégories sociales:

${ }^{27}$ Calvet, 1993b, page 90 .

"Mlid. pages 909\%.

"Plus précisément une conmnunau té des locuteurs inpose sa langue sur une conınunaute qui par des raisons politiques est subordonnee. 
les commerçants, les juristes, etc....Parallèlement, et pour les mêmes raisons d'intérêt et de nécessité, un autre groupe social va apprendre la langue dominante: celui des domestiques que l'envahisseur recruté sur place. Enfin, dans les situations où les arts et les lettres vivent du pouvoir l'expression adopte aussi cette langue dominante. Ce premier stade est donc le résultat d'une situation économique : la langue dominante est adoptée para ceux qui, sur place, sont proches du pouvoir ou le représentent, et par ceux qui traitent avec lui (gros commerçants ou domestiques). Ce bilinguisme ne concerne bien entendu qu'un petit groupe d'individus : l'immense majorité de la population demeure monolingue. ${ }^{30}$

Mais il y a aussi une sorte d'action sine qua non réciproque. Au début, quelques éléments du groupe envahisseur prennent contact avec les victimes en apprenant la (ou les) langue(s) dominée(s) afin de mieux 'apprivoiser' la population. Cet échange linguistique, dans notre contexte colombien a permis d'enrichir la langue dominante, l'espagnol, avant la disparition de quelques langues indigènes . ${ }^{31}$

Après quelques années, la suprématie de la langue espagnole avait dépassée les limites géographiques de la péninsule et s'est étendue en Europe et en Amérique possédant quelques traits représentatifs : prestige universel, correction linguistique, supériorité face aux langues indigènes (celles-ci étaient un obstacle pour la diffusion du christianisme) et diffusion d'une culture qui obéissait aux lois divines. Les langues indigènes et par conséquent les indiens, étaient considérées comme des barbares parce qu'ils ne connaissaient ni le dieu chrétien ni la langue espagnole. C'est pour cela que l'on a prétendu anéantir l'usage des langues indigènes pour éviter la décolonisation de la part des criollos et des propres indiens. Cependant, ces déclarations puristes, colonialistes et absurdes n'ont pas pu nuire au contact de l'espagnol péninsulaire avec la réalité du Nouveau Monde et l'Américanisme a, enfin vu le jour ! ${ }^{32}$ C'est pour cela qu'en Colombie on parle une variété de l'espagnol de l'Amérique, diversifié en patois régionaux dont les faibles différences structurales permettent une compréhension entre tous les usagers.

La force impérialiste de l'espagnol en Colombie lui donne le statut de langue officielle et nationale (c.-à-d. dominante). Ce qui nous intéresse dans le cas colombien, c'est avant tout son caractère néocolonialiste. Les langues indigènes et les créoles sont des langues et des communautés subordonnées à l'idéologie espagnole qui a stimulé la création d'une pseudoscience dénommée "Hispanistique " ${ }^{33}$. La Colombie a oublié l'une des versions de l'histoire de l'Amérique. C'est parce que sous le joug espagnol la Colombie est décrite comme "el Reino de la República de la Esclavolombia " Pour arriver à des précisions scientifiques, la linguistique ne devrait pas être une science militante, voire engagée. ${ }^{34}$ En d'autres termes, on peut contester l'imposition coloniale en faisant appel aux outils théoriques de la science et en demeurant dans l'état de l'objectivité logique.

2.2 Politique linguistique, planification linguistique, glottopolitique, écolinguistique

Pour mieux comprendre ce qui se passe dans la réalité communicative colombienne, nous allons définir quatre syntagmes qui sont profondément liés quand il s'agit d'expliquer le processus d'aménagement linguistique.

Selon Calvet $(1993 \mathrm{~b}, 111)$, la politique linguistique est un ensemble de choix conscients

\footnotetext{
"Calvet, 1974,

"Triana y Antorveza, 1987

"Ce que lon denomme conme le contact des langues.

"C'est la déclaration de Gutiérrez Girardot: ¿Qué es la hispanistica! ¿t hna ciencia o una iglesia?; O una arrogante miopia! Su anıpulosidad hinnica es extrenaadanente pern isiva.

${ }^{4}$ C'est la theise gue M. Rico defendait.
} 
concernant les rapports entre langue(s) et vie sociale. De plus, la planification linguistique est la mise en pratique concrète d'une politique linguistique, le passage à l'acte en quelque sorte. La glottopolitique, en termes de Marcellesi et Guespin (1986), est défini de la manière suivante :

Le concept de glottopolitique rend compte d'un axe vertical liant le fait normatif ou antinormatif apparemment le plus insignifiant aux faits les plus saillants de politique de la langue. Il couvre aussi un terrain horizontal beaucoup plus vaste que celui que couvre la notion de politique de la langue, car la novation glottopolitique n'est pas toujours perçue en tant que telle: toute décision modifiant les rapports sociaux est, du point de vue du linguiste, une décision glottopolitique [...] La mesure n'est certes pas une décision de politique de la langue, mais elle comporte une importante incidence glottopolitique : elle concerne la mise en discours de l'économie, des rapports de production, $d u$ monde du travail. Toute mesure qui affecte la répartition sociale de la parole, même si son objectif n'est pas langagier, intéresse la situation glottopolitique. Le terme glottopolitique peut être utilisé à deux fins : à la fois pour l'évocation des pratiques et pour la désignation de l'analyse; la glottopolitique est donc à la fois une pratique sociale, à laquelle nul n'échappe (on "fait de la glottopolitique sans le savoir ", qu'on soit simple citoyen ou ministre de l'économie), et elle a vocation à devenir une discipline de recherche, une branche aujourd'hui nécessaire de la sociolinguistique.

Pour ce qui est de l'écologie linguistique, on va la définir comme une approche qui a pour ambition d'intégrer une analyse complexe du langage en donnant les moyens a la sociolinguistique de rassembler l'ensemble des niveaux linguistiques et de mettre au jour les conditions sociales, mais aussi cognitives et environnementales de leur production. Ainsi, l'approche écologique semble vouloir accorder autant d'importance aux conditions dites extralinguistiques qu'aux conditions internes (intra-individuelles) dans une perspective différente de celle de la sociolinguistique traditionnelle ou des théories biologiques linguistiques récentes. Les représentants les plus connus sont : Haugen, Calvet, Halliday, Lechevrel, Makkai, Mühlhäusler, Bastardas Boada, etc.

\section{La Politique linguistique colombienne}

L'Etat colombien a proposé une politique linguistique (sans l'appui sociolinguistique voire écolinguistique) dont l'objectif c'est préparer les apprenants pour la maîtrise de l'anglais. Il ne s'agit pas d'une éducation en deux langues (le projet bilingue espagnol-anglais). Il s'agit de faire apprendre à l'apprenant la langue anglaise comme il a acquis sa première langue. Cependant, les résultats sont négatifs en ce qui concerne la maîtrise naturelle et authentique de ce code. De plus, on a écarté (voire on a effacé) les langues indigènes et les créoles qui font partie de la réalité sociolinguistique colombienne de la même manière que les autres langues étrangères telles le français, le portugais, l'italien etc.

\subsection{Les langues officielles colombiennes}

Quand on parle de politique linguistique on fait référence aux liens étroits entre l'État et la langue. L'État est déterminé par une forte unité nationale et une unité linguistique qui est issue des caractères de la langue instrument de pouvoir, instrument de communication véhiculaire, point de convergence entre les lois sociales et les lois linguistiques.

Bien que la langue joue un rôle privilégié dans les limites de l'Etat, les aspects politiques deviennent des marquants 'décideurs' en ce qui concerne l'unité. Selon Calvet ${ }^{35}$ la politique linguistique est " l'ensemble des choix conscients effectués dans le domaine des rapports entre langue et vie sociale." En d'autres termes, c'est l'Etat qui délimitera l'action de la langue dans la vie nationale et vice-versa. Prenons comme exemple notre cas colombien. La réforme de la 
Constitution Politique en 1991 a déclenché des changements administratifs pour les langues. On a reconnu la multiculturalite $[. .$.$] On a$ renforcé le rôle privilégié de l'espagnol, c'est-àdire comme langue officielle et les langues indigènes et créoles sont devenues officielles dans leurs territoires. Cela veut dire que la conservation de langues ethniques apparaît plus clairement comme un but utopique à atteindre. D'autres articles de la Constitution Politique s'en occupent :

ARTICULO 68. [...] Los integrantes de grupos étnicos tendrán derecho a una formación que respete y desarrolle su identidad cultural.

ARTICULO 70. [...] La cultura en sus diversas manifestaciones es fundamento de la nacionalidad. El Estado reconoce la igualdad y dignidad de todas las que conviven en el pais.

Les articles que l'on vient de citer, peuvent être considérés comme une esquisse de politique linguistique en Colombie. Cette politique linguistique représente une fonction symbolique malgré la présence indirecte d'une planification linguistique. Cela nous amène à considérer la politique linguistique colombienne comme le point de rencontre d'une fonction véhiculaire et d'une fonction grégaire,

Dans certains cas, c'est la même langue qui aura sa forme véhiculaire, celle que l'on parle dans la communication publique vers le plus grand nombre, et sa forme grégaire, celle qui limite la communication au plus petit nombre, à l'intimité ; dans d'autres cas, ces deux fonctions seront assumées par des langues différentes : mais toute politique sera confrontée à ces fonctions et à ces conflits qu'elles entretiennent éventuellement.

En ce qui concerne l'enseignement des langues étrangères, la réforme de la Constitution
Politique en 1991 a déclenché la création des nouvelles lois. La Loi 115 de 1994 (connue sous le nom de Loi général d'éducation) avec ses amendements correspondants et ses dispositions nouvelles, débute par divers points (i.e. articles):

c) El desarrollo de las habilidades comunicativas básicas para leer, comprender, escribir, escuchar, hablar y expresarse correctamente en lengua castellana y también en la lengua materna, en el caso de los grupos étnicos con tradición lingüistica propia, asi como el fomento de la afición por la lectura;

m) La adquisición de elementos de conversación y de lectura al menos en una lengua extranjera;

Cependant, à notre avis, la politique linguistique a échoué a cause de quatre raisons politiques : a) l'espagnol nuit à l'expansion des autres langues en raison de sa fonction dominante, b) le choix de l'anglais comme langue étrangère dominante, c) l'absence des conditions matérielles idéales pour mener à bien un tel projet d'intégration culturelle national et d) la mondialisation.

Sous l'optique de la sociolinguistique, le plurilinguisme colombien et la planification linguistique sont deux notions-réalités qui reflètent l'idéologie dépendante d'un pays sous la trilogie Etat- Nation- Langue, le cas de la Colombie illustre précisément l'action sur les langues. On a, d'une part, modifié le statut des langues ethniques et d'autre part, on a renforcé le statut dominant de l'espagnol. Cela relève d'une décision glottopolitique dans la mesure où l'on vise à transformer les rapports entre les langues en privilégiant une forme linguistique sur une autre.

La communauté exotique (dont les locuteurs des langues indigènes et créoles font partie) colombienne ne forme pas un ensemble sociolinguistique indépendant. Elle vit dispersée

35 19931, page 111. 
dans un pays immense qui veut se définir comme un Etat bilingue voire multilingue, c'està-dire, comme un pays dans lequel la langue espagnole et les langues indigènes et créoles auraient une reconnaissance officielle identique et lequel accorderait des droits légaux aux citoyens possédant l'une ou l'autre langue. Cependant, la réalité linguistique indique l'imposition de la langue espagnole et l'effacement progressif des langues autochtones. Si la Colombie est depuis longtemps multilingue, c'est le pays seul qui est multilingue (multilinguisme social). Les locuteurs colombiens, eux, ne sont en règle générale pas bilingues ou multilingues. Les seuls à être naturellement tous bilingues ou multilingues sont les locuteurs indigènes, tout au moins les adultes, dont beaucoup parlent ou comprennent en outre d'autres langues indigènes et l'espagnol.

\subsection{La place de l'anglais}

A cause de la mondialisation, la Colombie dispose d'un ensemble de mesures administratives et juridiques adoptées en vue de promouvoir l'anglais sur le territoire national. Le gouvernement colombien a crée des lois qui visent à faire de l'anglais non seulement la première langue étrangère des locuteurs colombiens mais aussi la langue commune dans les sphères de la vie publique à coté de l'espagnol. En d'autres termes, la politique linguistique a donc été instituée pour augmenter la force d'attraction de l'anglais, perceptible dans les villes les plus représentatives du pays : Bogotá. Medellín, Cali, Barranquilla, Bucaramanga, Armenia, Neiva etc.

Les linguistes ont conscience de la diversité linguistique et de l'influence de plus en plus marquée des pratiques langagières sur la société colombienne. Ce phénomène a incité, très tôt, les collectivités académiques à recourir à des outils d'organisation sociolinguistiques, non seulement pour s'approprier ou maîtriser l'usage de leurs propres langues ou variétés de langues, mais aussi pour tenter d'ajuster les relations interlinguistiques entre les groupes et les individus qui utilisaient des langues différentes à l'intérieur d'un espace social donné. La coexistence de groupes linguistiques sur un même territoire géographique est une réalité sociale qui s'est maintenant répandue et qui pose des problèmes sociolinguistiques de plus en plus emmerdants. Aujourd'hui, l'accroissement du plurilinguisme et de la concurrence linguistique au sein du territoire mondial délimite de nouveaux espaces linguistiques transnationaux, où l'on observe des tentatives officielles pour aménager les rapports entre les langues. Les situations et les dynamiques sociolinguistiques sont de plus en plus diversifiées à l'intérieur de nos sociétés modernes et elles obligent l'État à intervenir (souvent par des politiques ou des lois linguistiques) pour tenter d'aménager les rapports entre les groupes linguistiques en présence au sein d'un territoire géopolitique donné.

Adopter l'anglais comme langue véhiculaire n'est pas avant tout un acte linguistique mais un geste glottopolitique. Il s'agit de faire une intervention sociolinguistique voire écolinguistique capable de refléter la réalité linguistique du pays. Maintenant, nous devons poser deux questions : (i) Comment esquisser et mettre en ouvre un aménagement linguistique en Colombie? (ii) Quelles devraient être, à présent, les langues natives et étrangères cultivées en Colombie pour mener à bien l'aménagement sociolinguistique?

Il ne s'agit pas d'un débat de rhétorique. Les choix des langues relève d'une décision linguistique naturelle et spontanée étayée par des études sociolinguistiques sérieux. On peut clore cette réflexion en citant Hagège $(1995,22)$ :

*Or le plurilinguisme sera un besoin professionnel dans le vaste marché en construction. Et pour ceux qui ont l'amour des mots, il sera un moyen sur d'approfondir, para la comparaison, leur connaissance de leur langue maternelle.» 


\section{Bibliographie}

Achard P. 1995. "Formation discursive, dialogisme et sociologie ". Langages, $117: 82-95$.

Boyer H. 1996b. Sociolinguistique : territoire et objets. Neuchâtel, Delachaux et Niestlé.

Calvet L-J. 1974. Linguistique et colonialisme. Paris, Payot.

Calvet L-J. 1981. Les langues véhiculaires. Paris, PUF. Col. Que sais-je?

Calvet L-J. 1987. La guerre des langues. Paris, Payot. Calvet L-J. 1993b. La sociolinguistique. Paris, PUF. Col. Que sais-je?

Gadet, F. 1990. Saussure, une science de la langue. Paris, PUF.

Gardin B. et Marcellesi J-B. 1980. Sociolinguistique : approches, theories, pratiques. 2 vols. Parais, PUF.

Hagège C. 1995. " L'exigence du plurilinguisme "La paix pour les langues. Paris, Le Monde Bilingue.

Mahecha MA. 1999. Epistémologie de la sociolinguistique. Inédit.
Marcellesi J-B et Gardin B. 1974. Introduction à la sociolinguistique. La linguistique sociale. Paris, Larousse. Marcellesi J-B et Guespin L. 1986. * Pour la glottopolitique* Langages 83 : 5-34.

Milner J-C 1989. Introduction à une science du langage. Paris, Editions du Seuil.

Minaudier J-P. 1992. Histoire de la Colombie. De la conquête à nos jours. Paris, Editions L'Harmattan.

Montes JJ. 1995. Dialectologia general e hispanoamericana. Bogotá, Instituto Caro y Cuervo.

Rico A. 1981. Problèmes sociolinguistiques de la société colombienne. Mémoire de maitrise présenté et soutenu à l'Université de Grenoble II. Sous la direction de Mme Louise Dabène.

Théban L. 1968. " Géographie linguistique, typologie sociolinguistique "Revre Romaine de Linguistique XII-6 : 659. 663.

Triana y Antorveza H. 1987. Las lenguas indigenas en el Nuevo Reino de Granada. Bogotá, Instituto Caro y Cuervo, Biblioteca Ezequiel Uricoechea. 